\title{
Impurity and quaternions in nonrelativistic scattering from a quantum memory
}

\author{
Dionisios Margetis ${ }^{1,2}$ and Manoussos G Grillakis ${ }^{1}$ \\ ${ }^{1}$ Department of Mathematics, University of Maryland, College Park, MD 20742, USA \\ ${ }^{2}$ Institute for Physical Science and Technology, University of Maryland, College Park, \\ MD 20742, USA \\ E-mail: dio@math.umd.edu and mng@math.umd.edu
}

Received 28 August 2007, in final form 3 December 2007

Published 29 January 2008

Online at stacks.iop.org/JPhysA/41/065307

\begin{abstract}
Models of quantum computing rely on transformations of the states of a quantum memory. We study mathematical aspects of a model proposed by Wu in which the memory state is changed via the scattering of incoming particles. This operation causes the memory content to deviate from a pure state, i.e. induces impurity. For nonrelativistic particles scattered from a two-state memory and sufficiently general interaction potentials in $(1+1)$ dimensions, we express impurity in terms of quaternionic commutators. In this context, pure memory states correspond to null hyperbolic quaternions. In the case with point interactions, the scattering process amounts to appropriate rotations of quaternions in the frequency domain. Our work complements previous analyses by Margetis and Myers (2006 J. Phys. A 39 11567).
\end{abstract}

PACS numbers: 03.65.-w, 03.67.-a, 03.65.Nk, 03.65.Yz, 03.67.Lx, 03.65.Pm

\section{Introduction}

Ideally, quantum computations are performed via transforming pure states of a physical system called 'quantum memory' to other pure states (see, e.g., [1-6]). In this context, memory states transform unitarily. In most systems pure states may degrade to mixed states. This phenomenon amounts to decoherence. A well-known kind of decoherence is caused by extraneous influences unrelated to memory operations [6, 7].

Recently, Wu [8-10] introduced spatial variables in quantum computations by viewing the quantum memory as a scatterer: incoming particles are scattered from the memory and change its content. In this setting, unitary transforms apply to the combined system of memory and particles. The memory states do not transform unitarily unless the incoming signal is 'admissible'. In one-space dimension, single-frequency waves are admissible [9]. 
In practice, however, incoming signals are pulses of finite duration. Thus, their use leads to additional decoherence, which we term 'impurity'. This kind of decoherence is connected specifically to memory operations, as was first discussed in [9]. The impurity of a two-state memory was analyzed via the relativistic [9] and nonrelativistic [11] Schrödinger equations. In [11], the memory is allowed to interact with incoming particles only at one point by the use of the pseudo-potential derived in [8].

In the present paper, we extend the nonrelativistic formulation of [11] to reasonably general interaction potentials in one-space dimension. Our starting point is to model the interaction potential as an imaginary quaternion [12-15]. In this formalism, the impurity measure of [11] is expressed in terms of a norm that depends on quaternionic commutators (see proposition I of section 2.2). In this context, pure states correspond to null hyperbolic quaternions [16].

For point interactions, scattering from the memory admits a geometric interpretation in our framework: during the scattering process appropriately defined quaternions are rotated in the frequency domain. This simple picture motivates our approach, offers additional insight into properties of the impurity measure used in [11], and is amenable to computations. Our formalism, which aims to describe impurity within the traditional setting of quantum mechanics, is distinct from the known notion of 'quaternionic quantum mechanics' discussed in [17].

The analysis of the overall memory transformation, i.e. the detailed change of the memory state by scattering, lies beyond our present scope. Aspects of this transformation are described extensively in $[8,9]$. Noteworthy is the possibility of constructing an incoming wavefunction and interaction potential that always leave the memory content undistorted, with zero impurity. An example of this case for point interactions is mentioned in section 4.1. Such cases are viewed as too special for our purposes and therefore are not studied any further in this paper.

Our formulation in terms of quaternions is an application of the correspondence between quantum memory operations and special relativity pointed out in $[18,19]$. This correspondence relies on an isomorphism between Hermitian $2 \times 2$ matrices and the four-dimensional space $\mathrm{R}^{4}$. Notably, the work in $[18,19]$ is concerned with the measurement of pure states and does not address issues of impurity.

Scattering as an inherent mechanism of decoherence has been studied in the context of various physical systems including electrons and quantum dots interacting with phonons, e.g. $[20,21]$. In these settings use is made of perturbation theory: matrix representations of the interaction in momentum states are invoked without direct recourse to the time-dependent Schrödinger equation. Our approach is non-perturbative, based on solving the time-dependent Schrödinger equation with two coupled channels.

Our paper is organized as follows. In section 2, we formulate the problem of impurity for a two-state quantum memory as a scattering problem with two coupled channels and general interaction potential in one-space dimension: in section 2.1 we formulate the equations of motion by treating a local interaction potential as a quaternion; in section 2.2 , we express the time evolution of the impurity measure used in [11] in terms of commutators of quaternions; and in section 2.3, we describe an extension of this formulation to nonlocal interaction potentials. In section 3, we describe the general solution by invoking discrete schemes for amplitudes of suitable Fourier transforms in time. In section 4, we revisit the case with point interactions by use of the present formalism: in section 4.1 we focus on even wavefunctions; and in section 4.2 we treat odd wavefunctions. In section 5 , we summarize our results and discuss open problems. Throughout the analysis we apply units with $\hbar^{2} /(2 m)=1$ where $m$ is the particle mass and denote quaternions by boldface symbols distinct from vectors. 


\section{Formulation}

In this section, we describe the equations of motion for a general interaction potential. Subsequently, we derive an explicit formula for the impurity used in [11].

\subsection{Equations of motion}

For a two-state quantum memory [8], the field of the particle-memory system is the $2 \times 1$ (column) vector

$$
\vec{\psi}(x, t)=\left[\begin{array}{l}
\psi_{1}(x, t) \\
\psi_{2}(x, t)
\end{array}\right] \quad-\infty<x, \quad t<+\infty,
$$

where $\psi_{j}(x, t)(j=1,2)$ are scalar, square integrable functions in $(1+1)$ dimensions. The vector field $\vec{\psi}$ solves the Schrödinger equation,

$$
\mathrm{i} \partial_{t} \vec{\psi}=-\partial_{x}^{2} \vec{\psi}-\mathrm{iq}(x) \vec{\psi},
$$

where $-\mathbf{i q}(x)$ represents the interaction potential and $\mathbf{q}$ is a $2 \times 2$ skew-adjoint matrix (see (6)).

The $\mathbf{q}$ of (2) is written as

$$
\mathbf{q}(x)=\sum_{a} g^{a}(x) \breve{\sigma}_{a}, \quad a=1,2,3,
$$

which we call an 'imaginary quaternion' [12]; $g^{a}(x)$ are given real functions, $\breve{\sigma}_{a}:=\mathrm{i} \sigma_{a}$, and $\sigma_{\mu}(\mu=0,1,2,3)$ are the Pauli matrices with the usual convention $\sigma_{0}=\mathbf{1}$,

$$
\sigma_{1}=\left[\begin{array}{cc}
0 & 1 \\
1 & 0
\end{array}\right], \quad \sigma_{2}=\left[\begin{array}{cc}
0 & -\mathrm{i} \\
\mathrm{i} & 0
\end{array}\right], \quad \sigma_{3}=\left[\begin{array}{cc}
1 & 0 \\
0 & -1
\end{array}\right] .
$$

So, $\breve{\sigma}_{a}$ have the following properties:

$$
\begin{aligned}
& \breve{\sigma}_{a}^{\dagger}=-\breve{\sigma}_{a}, \quad \breve{\sigma}_{a}^{2}=-\mathbf{1}, \\
& \breve{\sigma}_{a} \breve{\sigma}_{b}=-\sum_{c} \epsilon_{a b c} \breve{\sigma}_{c} ; \quad a \neq b, \quad a, b, c=1,2,3,
\end{aligned}
$$

where $\sigma^{\dagger}$ denotes the conjugate transpose (Hermitian conjugate) of $\sigma$, and $\epsilon_{a b c}$ is the LeviCivita symbol. Hence, we ensure that

$$
\mathbf{q}^{\dagger}(x)=-\mathbf{q}(x) \text {. }
$$

A simplified form of (2) is

$$
\mathrm{i} \partial_{t} \vec{\psi}=-\partial_{x}^{2} \vec{\psi}-\mathrm{i}\left[g_{e}(x) \mathbf{u}_{e}+g_{o}(x) \mathbf{u}_{o}\right] \vec{\psi},
$$

where $\mathbf{u}_{e, o}$ are unit-length imaginary quaternions and $g_{e, o}(x)$ are even and odd functions, respectively.

We note in passing that the 'discrete version' of (2) reads

$$
\mathrm{i} \partial_{t} \vec{\psi}=-\partial_{x}^{2} \vec{\psi}-\sum_{j} \mathrm{iq}_{j} \delta\left(x-x_{j}\right) \vec{\psi}
$$

where $\left\{\mathbf{q}_{j}=\mathbf{q}\left(x_{j}\right)\right\}$ ( $j$ : integer) is a sequence of imaginary quaternions. By convolution of (8) with an appropriate kernel and for sufficiently dense partition $\left\{x_{j}\right\}$, the resulting solution can be arbitrarily close to the solution of (2). Because (8) is amenable to numerical computations, we discuss the relevant solutions in detail in section 3 . 
For later convenience, we introduce the Fourier transform in time of $\vec{\psi}(x, t)$ by assuming that this signal contains only positive frequencies. With the definition

$$
\vec{\psi}(x, t)=\int_{0}^{+\infty} \frac{\mathrm{d} \omega}{2 \pi} \mathrm{e}^{-\mathrm{i} \omega t} \vec{\phi}(x, \omega),
$$

the equation of motion (2) transforms to

$$
\omega \vec{\phi}(x, \omega)=-\partial_{x}^{2} \vec{\phi}(x, \omega)-\mathrm{iq}(x) \vec{\phi}(x, \omega) .
$$

\subsection{Impurity}

Next, we analyze impurity on the basis of (2). The reduced density matrix $\rho(t)$ for the memory is the $2 \times 2$ matrix [11]

$$
\rho(t):=\int_{-\infty}^{+\infty} \mathrm{d} x \vec{\psi}(x, t) \vec{\psi}^{\dagger}(x, t),
$$

which is obtained by tracing out the spatial variables. Of particular interest is the limit

$$
\mathbf{M}:=\lim _{t \rightarrow+\infty} \rho(t),
$$

which is connected to the final memory state. For vanishing impurity, $\mathbf{M}^{2}=\mathbf{M}$ and $\operatorname{tr}\left(\mathbf{M}^{2}\right)=1$. In [11], the impurity measure is defined by

$$
\operatorname{Imp}(\mathbf{M}):=\left[1-\operatorname{tr}\left(\mathbf{M}^{2}\right)\right]^{1 / 2} \text {. }
$$

In this subsection, we describe the $\mathbf{M}$ of (12). For this purpose, we form the density matrix

$$
\begin{aligned}
\mathbf{Q}(x, t) & :=\vec{\psi} \vec{\psi}^{\dagger}=\left[\begin{array}{ll}
\psi_{1} \psi_{1}^{*} & \psi_{1} \psi_{2}^{*} \\
\psi_{2} \psi_{1}^{*} & \psi_{2} \psi_{2}^{*}
\end{array}\right] \\
& =-\mathrm{i}\left(\mathrm{i}^{0} \sigma_{0}+\sum_{a} r^{a} \breve{\sigma}_{a}\right)=:-\mathrm{i} \mathbf{r}(x, t),
\end{aligned}
$$

where $\psi_{j}^{*}$ is the complex conjugate of $\psi_{j}$, and the coefficients $r^{\mu}(\mu=0,1,2,3)$ are

$$
\begin{aligned}
r^{0} & =\frac{1}{2}\left(\left|\psi_{1}\right|^{2}+\left|\psi_{2}\right|^{2}\right), & r^{1} & =\frac{1}{2}\left(\psi_{1} \psi_{2}^{*}+\psi_{1}^{*} \psi_{2}\right), \\
r^{2} & =\frac{1}{2 \mathrm{i}}\left(\psi_{1}^{*} \psi_{2}-\psi_{1} \psi_{2}^{*}\right), & r^{3} & =\frac{1}{2}\left(\left|\psi_{1}\right|^{2}-\left|\psi_{2}^{*}\right|^{2}\right) .
\end{aligned}
$$

It follows that

$$
\begin{aligned}
& \rho(t)=-\mathrm{i} \int_{-\infty}^{+\infty} \mathrm{d} x \mathbf{r}(x, t)=:-\mathrm{im}(t), \quad \mathbf{m}(t):=\int_{-\infty}^{+\infty} \mathrm{d} x \mathbf{r}(x, t), \\
& \mathbf{M}=-\mathrm{i} \lim _{t \rightarrow+\infty} \mathbf{m}(t)=:-\mathbf{i m}_{\text {out }} .
\end{aligned}
$$

An important remark is in order. With $\mathbf{r}=\mathrm{i} r^{0} \sigma_{0}+\sum_{a} r^{a} \breve{\sigma}_{a}$ by (14), the dual of $\mathbf{r}$ is defined by

$$
\mathbf{r}^{\mathrm{d}}:=\mathrm{i} r^{0} \sigma_{0}-\sum_{a} r^{a} \breve{\sigma}_{a} .
$$

By virtue of (15), we have

$$
\mathbf{r r}^{\mathrm{d}}=:\langle\mathbf{r}, \mathbf{r}\rangle \equiv 0,
$$


i.e., $\mathbf{r}$ is identified with a null hyperbolic quaternion $[16,18] ;\langle\cdot, \cdot\rangle$ is the Minkowski inner product by identifying $\mathbf{r}$ with the 4-vector $\left(r^{0}, r^{1}, r^{2}, r^{3}\right)$. Note that $\mathbf{m}$ in (16) is an integral of null quaternions. Because of the convexity of the characteristic cone [14], this integral produces either a timelike $\left(\mathbf{m m}^{\mathrm{d}}<0\right)$ or a null $\left(\mathbf{m m}^{\mathrm{d}}=0\right)$ quaternion. This property ensures that the $1-\operatorname{tr}\left(\mathbf{M}^{2}\right)$ in (13) is non-negative (see appendix).

We now derive an equation of motion for $\mathbf{Q}(x, t)$. With the system Hamiltonian $\mathbf{H}:=-\mathbf{1} \partial_{x}^{2}-\mathbf{i q}(x),(2)$ becomes $\mathrm{i} \partial_{t} \vec{\psi}=\mathbf{H} \vec{\psi}$. By (3), we readily obtain

$$
\partial_{t} \mathbf{Q}=-\mathrm{i}\left(\mathbf{H}_{\rightarrow} \vec{\psi}\right) \vec{\psi}^{\dagger}+\mathrm{i} \vec{\psi}\left(\vec{\psi}_{\leftarrow}^{\dagger} \mathbf{H}\right)
$$

where $\vec{\psi}_{\leftarrow}^{\dagger} \mathbf{H}$ denotes the action of $\mathbf{H}$ on $\vec{\psi}^{\dagger}$ from the left. Equation (20) is recast to the conservation law

$$
\partial_{t} \mathbf{Q}+\partial_{x} \mathbf{P}=[\mathbf{Q}(x, t), \mathbf{q}(x, t)],
$$

where $[\mathbf{Q}, \mathbf{q}]:=\mathbf{Q q}-\mathbf{q} \mathbf{Q}$ is the commutator of two quaternions and $\mathbf{P}$ is the 'flux matrix'

$$
\mathbf{P}=\frac{1}{\mathrm{i}}\left[\left(\partial_{x} \vec{\psi}\right) \vec{\psi}^{\dagger}-\vec{\psi}\left(\partial_{x} \vec{\psi}^{\dagger}\right)\right]
$$

By (16) and (17), we need to integrate (20) over space and then time. Accordingly, we obtain

$$
\Delta \mathbf{m}:=\mathbf{m}_{\text {out }}-\mathbf{m}_{\text {in }}=\int_{-\infty}^{+\infty} \int_{-\infty}^{+\infty} \mathrm{d} t \mathrm{~d} x[\mathbf{r}(x, t), \mathbf{q}(x)],
$$

where $\mathbf{m}_{\text {in }}:=\lim _{t \rightarrow-\infty} \mathbf{m}(t)$ is given. Consequently,

$$
\mathbf{M}=-\mathrm{i}\left(\mathbf{m}_{\text {in }}+\Delta \mathbf{m}\right) \text {. }
$$

It is convenient to rewrite $\Delta \mathbf{m}$ of (23) in terms of the appropriate Fourier transform. Plancherel's formula [22] and definition (14) give

$$
\int_{-\infty}^{+\infty} \mathrm{d} t \mathbf{r}(x, t)=\int_{0}^{+\infty} \frac{\mathrm{d} \omega}{2 \pi} \widetilde{\mathbf{r}}(x, \omega)
$$

where $\widetilde{\mathbf{r}}(x, \omega)$ is the quaternion corresponding to the frequency-domain density matrix $\vec{\phi}(x, \omega) \vec{\phi}^{\dagger}(x, \omega)$ by (9), i.e.,

$$
\widetilde{\mathbf{r}}(x, \omega):=\mathrm{i} \vec{\phi}(x, \omega) \vec{\phi}^{\dagger}(x, \omega),
$$

assuming that the signals have only positive-frequency content. Thus, we have the formula

$$
\Delta \mathbf{m}=\int_{0}^{+\infty} \frac{\mathrm{d} \omega}{2 \pi} \int_{-\infty}^{+\infty} \mathrm{d} x[\widetilde{\mathbf{r}}(x, \omega), \mathbf{q}(x)] .
$$

We note in passing that (27) can be formally generalized for higher space dimensions, where $\vec{\phi}(x, \omega)$ satisfies the vector Helmholtz equation.

We now restrict attention to pure initial memory states. The incoming vector field has the product form

$$
\vec{\psi}_{\text {in }} \sim \psi_{\text {in }}(x, t) \vec{s}_{\text {in }}, \quad t \rightarrow-\infty,
$$

where $\psi_{\text {in }}(x, t)$ is the incoming particle wavefunction and $\vec{s}_{\text {in }}$ is the initial memory state. For example, we have [11]

$$
\psi_{\text {in }}(x, t)=\int_{0}^{+\infty} \frac{\mathrm{d} \omega}{2 \pi} \mathrm{e}^{-\mathrm{i} \omega t-\mathrm{i} \sqrt{\omega}|x|} f(\omega) \cdot \begin{cases}1, & \text { even wave } \\ \operatorname{sg}(x), & \text { odd wave }\end{cases}
$$

where $\operatorname{sg}(x)$ is the usual sign function, i.e., $\operatorname{sg}(x)=1$ if $x>0, \operatorname{sg}(x)=-1$ if $x<0$ and $\operatorname{sg}(0)=0$. The incoming quaternion $\mathbf{m}_{\text {in }}$ is [11]

$$
\mathbf{m}_{\text {in }}=4 \int_{0}^{+\infty} \frac{\mathrm{d} \omega}{2 \pi}|f(\omega)|^{2} \sqrt{\omega}\left(\vec{s}_{\mathrm{in}} \vec{s}_{\mathrm{in}}^{\dagger}\right) .
$$


We view (30) as the general definition of $f(\omega)$ (without specifying any symmetry in $x$ ). By analogy with (19), $\mathbf{m}_{\text {in }}$ satisfies

$$
\mathbf{m}_{\text {in }} \mathbf{m}_{\text {in }}^{d}=0 .
$$

Similarly, the condition $\operatorname{tr}\left(\mathbf{M}^{2}\right)=1$ (pure final memory state) corresponds to $\mathbf{m}_{\text {out }} \mathbf{m}_{\text {out }}^{d}=0$. So, pure states are described by null hyperbolic quaternions. This mapping is one to one, as stated in proposition I below.

The frequency profile $|f(\omega)|^{2}$ in (30) is chosen so that $\mathbf{m}_{\text {out }}$ is as close to null as possible. We state the following proposition.

Proposition 1. The impurity measure (13) reads

$$
\operatorname{Imp}(\mathbf{M})=\sqrt{2\left|\left\langle\mathbf{m}_{\text {out }}, \mathbf{m}_{\text {out }}\right\rangle\right|}=\sqrt{2\left|2\left\langle\mathbf{m}_{\text {in }}, \Delta \mathbf{m}\right\rangle+\langle\Delta \mathbf{m}, \Delta \mathbf{m}\rangle\right|},
$$

where $\langle\cdot, \cdot\rangle$ is the Minkowski inner product defined in (19). (Thus, minimizing impurity is equivalent to minimizing the Minkowski norm corresponding to $\mathbf{m}_{\text {out.) }}$ In addition, zero impurity is equivalent to $\mathbf{m}_{\mathrm{out}}$ being a null hyperbolic quaternion.

A proof of (32) follows directly from the definition of $\mathbf{m}_{\text {out }}=\mathrm{i} \mathbf{M}$ and (13). We sketch the main steps here (for details see appendix). By the representation of $\mathbf{m}_{\text {out }}$ in terms of the Pauli matrices we find $\left|\left\langle\mathbf{m}_{\text {out }}, \mathbf{m}_{\text {out }}\right\rangle\right|=\operatorname{det} \mathbf{M}$. Recall that the impurity measure is $\operatorname{Imp}(\mathbf{M})=\sqrt{2(\operatorname{det} \mathbf{M})}[11]$, and for a pure initial state we have $\left\langle\mathbf{m}_{\text {in }}, \mathbf{m}_{\text {in }}\right\rangle=0$.

Proposition I shows that definition (13) for $\operatorname{Imp}(\mathbf{M})$ is a natural choice: the deviation from a pure state is expressed in terms of the 'length' of a hyperbolic quaternion. The impurity can be obtained from (32) combined with (27), (26) and (30) once $\vec{\phi}$ is known. The reader is referred to section 3 for details on $\vec{\phi}(x, \omega)$.

\subsection{Extension}

In the case with a nonlocal interaction potential [8], the equation of motion is

$$
\mathrm{i} \partial_{t} \vec{\psi}=-\partial_{x}^{2} \vec{\psi}-\int_{-\infty}^{+\infty} \mathrm{d} y \mathrm{iq}(x, y) \vec{\psi}(y, t) .
$$

The corresponding equation for $\mathbf{Q}=\vec{\psi} \vec{\psi}^{\dagger}$ is

$\partial_{t} \mathbf{Q}+\partial_{x} \mathbf{P}=\int_{-\infty}^{+\infty} \mathrm{d} y\left\{\vec{\psi}(x, t) \vec{\psi}^{\dagger}(y, t) \mathbf{q}(x, y)-\mathbf{q}(x, y) \vec{\psi}(y, t) \vec{\psi}^{\dagger}(x, t)\right\}$

If $\mathbf{q}(x, y)=\mathbf{q} V_{\alpha}(x) V_{\beta}(y)$ and $V_{\alpha, \beta}$ are scalar functions [8], integration of (34) yields

$$
\partial_{t} \mathbf{m}=\left[\mathbf{r}_{V}, \mathbf{q}\right]
$$

where the quaternion $\mathbf{r}_{V}$ is defined by

$$
\mathbf{r}_{V}=\int_{-\infty}^{+\infty} \mathrm{d} y V_{\beta}(y) \vec{\psi}(y, t) \int_{-\infty}^{+\infty} \mathrm{d} x V_{\alpha}(x) \vec{\psi}^{\dagger}(x, t)
$$

\section{General solution scheme}

In this section, we describe the Fourier transform $\vec{\phi}(x, \omega)$ of $\vec{\psi}(x, t)$ by solving (10). We start with (8), the discrete analogue of (2). The Fourier decomposition (9) reduces (8) to the form

$$
\omega \vec{\phi}(x, \omega)=-\partial_{x}^{2} \vec{\phi}(\omega, x)-\sum_{j} \mathrm{i} \delta\left(x-x_{j}\right) \mathbf{q}_{j} \vec{\phi}_{j}
$$


where $\vec{\phi}_{j}:=\vec{\phi}\left(x_{j}, \omega\right)$. The general solution of (37) is

$$
\vec{\phi}(x, \omega)=\vec{a}_{+}(\omega) \mathrm{e}^{\mathrm{i} \sqrt{\omega} x}+\vec{a}_{-}(\omega) \mathrm{e}^{-\mathrm{i} \sqrt{\omega} x}+\sum_{j} \mathrm{iq}_{j} \vec{\phi}_{j} G\left(x-x_{j}\right),
$$

where $\vec{a}_{ \pm}$are reasonably arbitrary vectors and $G(x, \omega)$ is the Green's function defined by

$$
G(x, \omega)=\frac{\mathrm{i}}{4 \sqrt{\omega}}\left(\mathrm{e}^{\mathrm{i} \sqrt{\omega}|x|}-\mathrm{e}^{-\mathrm{i} \sqrt{\omega}|x|}\right) .
$$

By inspection of (38) and (39), $\vec{\phi}$ reads

$$
\vec{\phi}(x, \omega)=\vec{d}_{+}(x, \omega) \mathrm{e}^{\mathrm{i} \sqrt{\omega} x}+\vec{d}_{-}(x, \omega) \mathrm{e}^{-\mathrm{i} \sqrt{\omega} x},
$$

where only the values $\vec{d}_{ \pm}\left(x_{j}, \omega\right)$ matter.

In principle, the coefficients $\vec{d}_{ \pm}$can be determined from the incoming wavefunction $\vec{\psi}_{\text {in }}$, formula (28). In the limit $t \rightarrow-\infty$ we have [11]

$$
\int_{0}^{+\infty} \frac{\mathrm{d} \omega}{2 \pi} \vec{d}_{ \pm}(x, \omega) \mathrm{e}^{ \pm \mathrm{i} \sqrt{\omega} x-\mathrm{i} \omega t} \sim \psi_{\text {in }}(x, t) \vec{s}_{\mathrm{in}}, \quad t \rightarrow-\infty,
$$

where the upper (lower) sign is taken for $x<0(x>0)$. In particular, we set $x \rightarrow \mp \infty$ [11]. So, the last relation gives $\vec{d}_{+}(x, \cdot)$ if $x<-M_{+}$and $\vec{d}_{-}(x, \cdot)$ if $x>M_{-}$for sufficiently large $M_{ \pm}$by use of the Fourier transform of $\psi_{\text {in }}$, e.g. (29). In the following, we find $\vec{\phi}(x, \cdot)$ everywhere via a scheme that determines $\vec{d}_{ \pm}\left(x_{j}, \omega\right)$.

Next, we derive equations for $\vec{d}_{ \pm, j}:=\vec{d}_{ \pm}\left(x_{j}, \omega\right)$ on the basis of (37). For this purpose, we introduce the four-component vectors

$$
\vec{d}(x, \omega):=\left[\begin{array}{l}
\vec{d}_{+} \\
\vec{d}_{-}
\end{array}\right], \quad \vec{a}(\omega):=\left[\begin{array}{l}
\vec{a}_{+} \\
\vec{a}_{-}
\end{array}\right] .
$$

Equation (38) reads

$$
\vec{d}_{j}=\vec{a}-\sum_{k} \operatorname{sg}(j-k) \Omega_{k} \vec{d}_{k},
$$

where $\vec{d}_{j}:=\vec{d}\left(x_{j}, \omega\right)$ and $\Omega_{j}$ is the $4 \times 4$ matrix

$$
\Omega_{j}:=\frac{1}{4 \sqrt{\omega}}\left[\begin{array}{cc}
\mathbf{q}_{j} & w_{j} \mathbf{q}_{j} \\
-w_{j}^{*} \mathbf{q}_{j} & -\mathbf{q}_{j}
\end{array}\right], \quad w_{j}:=\mathrm{e}^{-2 \mathrm{i} \sqrt{\omega} x} .
$$

By taking differences in (43) we find the equation

$$
\left(I+\Omega_{j+1}\right) \vec{d}_{j+1}=\left(I-\Omega_{j}\right) \vec{d}_{j},
$$

where $I$ is the $4 \times 4$ unit matrix. By the identity $\left(\Omega_{j}\right)^{2} \equiv 0$ we write (45) as

$$
\vec{d}_{j+1}=\left(I+\Omega_{j+1}\right)^{-1}\left(I-\Omega_{j}\right) \vec{d}_{j}=\left(I-\Omega_{j+1}\right)\left(I-\Omega_{j}\right) \vec{d}_{j} .
$$

Let us assume that $\mathbf{q}_{j}$ have finite range, i.e.,

$$
\mathbf{q}_{j} \equiv 0, \quad|j|>N,
$$

for some fixed positive integer $N$. Define

$$
\vec{d}_{\text {left }}:=\vec{d}_{j}, \quad j<-N, \quad \vec{d}_{\text {right }}:=\vec{d}_{j}, \quad j>N,
$$

which are constants. By introducing the $4 \times 4$ matrices

$$
R_{j}:=\left(I-\Omega_{j}\right) \prod_{k=-N}^{j-1}\left(I-2 \Omega_{k}\right), \quad R:=\prod_{k=-N}^{N}\left(I-2 \Omega_{k}\right),
$$


we derive the relations

$$
\vec{d}_{j}=R_{j} \vec{d}_{\text {left }}, \quad|j| \leqslant N, \quad \vec{d}_{\text {right }}=R \vec{d}_{\text {left }} .
$$

By the summation form (43) we obtain the formulae

$$
\vec{d}_{\text {left }}=\vec{a}+\sum_{j} \Omega_{j} R_{j} \vec{d}_{\text {left }}, \quad R \vec{d}_{\text {left }}=\vec{a}-\sum_{j} \Omega_{j} R_{j} \vec{d}_{\text {left }},
$$

by which we find the relations

$$
I-R=2 \sum_{j} \Omega_{j} R_{j}, \quad \vec{a}=\frac{1}{2}(I+R) \vec{d}_{\text {left }} .
$$

Thus, by (50), $\vec{d}_{\text {left }}$ alone suffices to yield $\vec{\phi}(x, \omega)$ in (38). With recourse to (41), the incoming wavefunction $\vec{\psi}_{\text {in }}$ furnishes immediately the $2 \times 2$ vectors $\vec{d}_{+, \text {left }}$ and $\vec{d}_{-, \text {right }}$. By writing

$$
R=:\left[\begin{array}{ll}
\check{\mathbf{R}}_{1} & \check{\mathbf{R}}_{2} \\
\check{\mathbf{R}}_{3} & \check{\mathbf{R}}_{4}
\end{array}\right]
$$

where $\check{\mathbf{R}}_{k}$ are $2 \times 2$ matrices, and using (50) we find

$$
\vec{d}_{-, \text {left }}=\check{\mathbf{R}}_{4}^{-1}\left(\vec{d}_{-, \text {right }}-\check{\mathbf{R}}_{3} \vec{d}_{+, \text {left }}\right) .
$$

For the sake of simplicity, we assume that $\check{\mathbf{R}}_{4}$ is non-singular. The last relation completes the calculation of the 4-vector $\vec{d}_{\text {left }}$. Thus, we arrive at the following statement.

Proposition 2. Equation (37), with $\mathbf{q}_{j} \equiv 0$ for $|j|>N$, is solved by (38) where $\vec{a}$ given by (52),

$$
\vec{\phi}_{j}=\vec{d}_{+, j} \mathrm{e}^{\mathrm{i} \sqrt{\omega} x_{j}}+\vec{d}_{-, j} \mathrm{e}^{-\mathrm{i} \sqrt{\omega} x_{j}}
$$

and $\vec{d}_{ \pm, j}$ are given by (50); $\vec{d}_{+, \text {left }}$ is determined by (41) and $\vec{d}_{-, \text {left }}$ is determined by (54).

The continuous analog of (43), which pertains to the solution of (10), is the Fredholm-type integral equation

$$
\left.\vec{d}(x, \omega)=\vec{a}(\omega)-\int_{-\infty}^{+\infty} \mathrm{d} y \operatorname{sg}(x-y) \Omega(y, \omega)\right) \vec{d}(y, \omega),
$$

where the $4 \times 4$ matrix $\Omega$ is

$$
\Omega(x, \omega)=\frac{1}{4 \sqrt{\omega}}\left[\begin{array}{cc}
\mathbf{q}(x) & w(x) \mathbf{q}(x) \\
-w^{*}(x) \mathbf{q}(x) & -\mathbf{q}(x)
\end{array}\right], \quad w(x)=\mathrm{e}^{-2 \mathrm{i} \sqrt{\omega} x} .
$$

Differentiation of (56) leads to the Dirac-type equation

$$
\partial_{x} \vec{d}(x, \omega)=-2 \Omega(x, \omega) \vec{d}(x, \omega) .
$$

Note that the scheme underlying proposition II corresponds to solving (56) by iterations. Because of the obvious connection of this scheme to the standard theory of integral equations [23], we do not discuss (56) any further in this paper. Once $\vec{\phi}(x, \omega)$ is known, $\operatorname{Imp}(\mathbf{M})$ can be calculated via proposition I in section 2.2. In the following section, we apply proposition II to a delta-function potential $[8,11]$.

The procedure of this section, which applies to the Schrödinger equation (2) with a local interaction, can be extended to nonlocal interactions, equation (33), but the algebra is more elaborate. In the following section, we consider the case where the kernel of the interaction becomes a suitable pseudo-potential $[8,11]$. 


\section{Point interaction}

In this section, we revisit the theory of [11] in the context of the present formalism, particularly of propositions I, II in sections 2.2, 3. For point interactions and a pure initial state of the memory, we calculate the impurity measure and show that the scattering amounts to rotations of quaternions in the frequency domain. We describe how a class of incoming finite-energy pulses can produce small impurity.

\subsection{Even wavefunctions}

First, we consider the Schrödinger equation

$$
\mathrm{i} \partial_{t} \vec{\psi}(x, t)=-\partial_{x}^{2} \vec{\psi}-\mathrm{iq} \delta(x) \vec{\psi}(0, t),
$$

by which the particle interacts with the memory at the origin. We set

$$
\mathbf{q}=g \mathbf{u}, \quad\|\mathbf{u}\|=1,
$$

i.e., $\mathbf{u}$ is a unit imaginary quaternion; $\|\mathbf{u}\|:=\sqrt{\langle\mathbf{u}, \mathbf{u}\rangle}$ in the Minkowski space. The incoming wavefunction is assumed to be the even part of (29) [11].

By virtue of (27) and (32), the impurity is measured in terms of the quaternionic commutator

$$
\Delta \mathbf{m}=\int_{0}^{+\infty} \frac{\mathrm{d} \omega}{2 \pi}[\widetilde{\mathbf{r}}(0, \omega), g \mathbf{u}]
$$

where $\widetilde{\mathbf{r}}(0, \omega)$ is

$$
\widetilde{\mathbf{r}}(0, \omega)=\mathrm{i} \vec{\phi}(0, \omega) \vec{\phi}^{\dagger}(0, \omega)
$$

We now apply the formalism of section 3, in particular proposition II. In the present situation we have $N=0$; the associated vector coefficients are $\vec{d}_{-1}, \vec{d}_{0}$ and $\vec{d}_{1}$. By (49) there is only one propagation matrix, i.e.,

$$
R_{0}=I-\Omega_{0}=\left[\begin{array}{cc}
\mathbf{1}-\frac{g}{4 \sqrt{\omega}} \mathbf{u} & -\frac{g}{4 \sqrt{\omega}} \mathbf{u} \\
\frac{g}{4 \sqrt{\omega}} \mathbf{u} & \mathbf{1}+\frac{g}{4 \sqrt{\omega}} \mathbf{u}
\end{array}\right]
$$

Thus,

$$
\vec{d}_{\text {left }}=\vec{d}_{-1}=\left(I+\Omega_{0}\right) \vec{d}_{0}, \quad \vec{d}_{\text {right }}=\vec{d}_{1}=\left(I-\Omega_{0}\right) \vec{d}_{0} .
$$

The introduction of the $2 \times 1$ vector $\vec{d}_{s}$ by

$$
\vec{d}_{0}=:\left[\begin{array}{l}
\vec{d}_{s} \\
\vec{d}_{s}
\end{array}\right]
$$

converts (64) to

$$
\vec{d}_{j}=\left[\begin{array}{l}
\vec{d}_{s} \\
\vec{d}_{s}
\end{array}\right]-\operatorname{sg}(j) \frac{g}{2 \sqrt{\omega}} \mathbf{u} \cdot\left[\begin{array}{c}
\vec{d}_{s} \\
-\vec{d}_{s}
\end{array}\right], \quad j=-1,0,1 .
$$

Vectors relevant to $\Delta \mathbf{m}$ of (61) are

$$
\vec{d}_{\text {in }}:=\vec{d}_{+, \text {left }}=\left(\mathbf{1}+\frac{g}{2 \sqrt{\omega}} \mathbf{u}\right) \vec{d}_{s}, \quad \vec{\phi}(0, \omega)=2 \vec{d}_{s} .
$$

By (61), the entanglement quaternion $\Delta \mathbf{m}$ reads

$$
\Delta \mathbf{m}=4 \int_{0}^{+\infty} \frac{\mathrm{d} \omega}{2 \pi}\left[\mathrm{i} \vec{d}_{s} \vec{d}_{s}^{\dagger}, g \mathbf{u}\right]=\int_{0}^{+\infty} \frac{\mathrm{d} \omega}{2 \pi} \frac{4 g}{p^{2}} \mathrm{e}^{-\theta \mathbf{u}}\left[\mathrm{i} \vec{d}_{\mathrm{in}} \vec{d}_{\mathrm{in}}^{\dagger}, \mathbf{u}\right] \mathrm{e}^{\theta \mathbf{u}}
$$


where we conveniently defined the quaternion

$$
\mathbf{p}(\omega):=\mathbf{1}+\frac{g}{2 \sqrt{\omega}} \mathbf{u}=: p \mathrm{e}^{\theta \mathbf{u}}
$$

and applied the convention that the magnitude and phase are

$$
p(\omega)=\sqrt{1+\frac{g^{2}}{4 \omega}}, \quad \theta(\omega)=\arctan \left(\frac{g}{2 \sqrt{\omega}}\right) .
$$

We now simplify (68) by observing that $\mathrm{d} \omega / \mathrm{d} \theta=-(4 / g) p^{2} \omega^{3 / 2}$ and

$$
\mathrm{i} \vec{d}_{\text {in }} \vec{d}_{\text {in }}^{\dagger}=|f(\omega)|^{2} \mathbf{t}_{\text {in }}, \quad \mathbf{t}_{\text {in }}:=\mathrm{i}\left(\vec{s}_{\text {in }} \vec{s}_{\text {in }}^{\dagger}\right),
$$

where $\vec{s}_{\text {in }}$ is introduced in (29). Furthermore, we apply the identity

$$
\frac{\mathrm{d}}{\mathrm{d} \theta}\left(\mathrm{e}^{-\theta \mathbf{u}} \mathbf{t} \mathrm{e}^{\theta \mathbf{u}}\right)=\mathrm{e}^{-\theta \mathbf{u}}[\mathbf{t}, \mathbf{u}] \mathrm{e}^{\theta \mathbf{u}}
$$

where the operation $\mathrm{e}^{-\theta \mathbf{u}} \mathbf{t} \mathrm{e}^{\theta \mathbf{u}}$ is a rotation which leaves the plane spanned by $\left\{\sigma_{0}, \mathbf{u}\right\}$ invariant. We can find two imaginary quaternions $\{\mathbf{v}, \mathbf{w}\}$ that are orthogonal to $\mathbf{u}$. Let $P_{\mathbf{u}}$ be the projection onto the space spanned by $\{\mathbf{v}, \mathbf{w}\}$. Equations (68)-(72) entail

$$
\Delta \mathbf{m}=-16 \int_{0}^{+\infty} \frac{\mathrm{d} \omega}{2 \pi} \omega^{3 / 2}|f(\omega)|^{2} \frac{\mathrm{d}}{\mathrm{d} \omega}\left\{\mathrm{e}^{-\theta \mathbf{u}}\left(P_{\mathbf{u}} \mathbf{t}_{\text {in }}\right) \mathrm{e}^{\theta \mathbf{u}}\right\} .
$$

Integration by parts yields

$$
\Delta \mathbf{m}=16 \int_{0}^{+\infty} \frac{\mathrm{d} \omega}{2 \pi} \mathrm{e}^{-\theta(\omega) \mathbf{u}}\left(P_{\mathbf{u}} \mathbf{t}_{\mathrm{in}}\right) \mathrm{e}^{\theta(\omega) \mathbf{u}}\left(\omega^{3 / 2}|f(\omega)|^{2}\right)^{\prime},
$$

where the prime denotes differentiation with respect to $\omega$.

A remark on (74) is in order. In the special case where $P_{\mathbf{u}} \mathbf{t}_{\text {in }}=0$, i.e. the incoming quaternion $\mathbf{t}_{\text {in }}$ is parallel to $\mathbf{u}$, the potential is not 'seen' by the particle and $\Delta \mathbf{m}=0$. Then, the memory is left at the same state producing zero impurity, $\operatorname{Imp}(\mathbf{M})=0$.

Example of small-impurity pulse. We proceed to describe how a class of incoming finiteenergy pulses can produce an arbitrarily small impurity. Such pulses have of course a narrow spectrum sufficiently localized at a single frequency.

A simple case of pulses with finite energy is described by

$$
(2 \pi)^{-1} \omega^{3 / 2}|f(\omega)|^{2}=H\left(\omega-\omega_{0}\right)-H\left(\omega-K \omega_{0}\right), \quad K>1, \quad \omega_{0}>0,
$$

where $H$ is the Heavyside function $\left(H^{\prime}(\omega)=\delta(\omega)\right)$ and $\left(\omega_{0}, K\right)$ are given parameters. The amplitude $|f(\omega)|^{2}$ is scaled by $4 \int_{0}^{+\infty} \mathrm{d} \omega|f|^{2} \sqrt{\omega}$ so that the total pulse energy is fixed to unity. This normalization will be carried out in the impurity measure $\operatorname{Imp}(\mathbf{M})$ below. In view of (74), we compute

$$
\Delta \mathbf{m}=16\left(\mathrm{e}^{-\theta_{1} \mathbf{u}} P_{\mathbf{u}} \mathbf{t}_{\text {in }} \mathrm{e}^{\theta_{1} \mathbf{u}}-\mathrm{e}^{-\theta_{2} \mathbf{u}} P_{\mathbf{u}} \mathbf{t}_{\text {in }} \mathrm{e}^{\theta_{2} \mathbf{u}}\right)
$$

where

$\theta_{1}=\arctan \left(\frac{g}{2 \sqrt{\omega_{0}}}\right), \quad \theta_{2}=\arctan \left(\frac{g}{2 \sqrt{K \omega_{0}}}\right) ; \quad 0<\theta_{2}<\theta_{1}<\frac{\pi}{2}$.

Note that the operation $\mathrm{e}^{-\theta \mathbf{u}} P_{\mathbf{u}} \mathbf{t}_{\text {in }} \mathrm{e}^{\theta}$ rotates $P_{\mathbf{u}} \mathbf{t}_{\text {in }}$ by $-2 \theta$.

With regard to $\mathbf{m}_{\text {in }}$, by (30) we compute

$$
\mathbf{m}_{\text {in }}=4\left(\int_{0}^{+\infty} \frac{\mathrm{d} \omega}{2 \pi}|f(\omega)|^{2} \sqrt{\omega}\right) \mathbf{t}_{\text {in }}=4 \ln (K) \mathbf{t}_{\text {in }} .
$$

10 
We note that $\Delta \mathbf{m}$ lies in the plane spanned by $\{\mathbf{v}, \mathbf{w}\}$ which is orthogonal to $\{\mathbf{1}, \mathbf{u}\}$. Substituting into (32) and normalizing properly we find that the impurity measure equals

$$
\operatorname{Imp}(\mathbf{M})=4 \sqrt{\frac{\left|4\left\{1-\cos \left(2 \theta_{1}-2 \theta_{2}\right)\right\}+\ln (K)\left\{\cos \left(2 \theta_{1}\right)-\cos \left(2 \theta_{2}\right)\right\}\right|}{(\ln K)^{2}}} .
$$

The right-hand side of this expression vanishes only for $K=1$, but becomes arbitrarily small if $K-1 \ll 1$. In this limit,

$$
\operatorname{Imp}(\mathbf{M}) \sim \sqrt{\frac{2}{3}} \frac{g /\left(2 \sqrt{\omega_{0}}\right)}{\omega_{0}+g^{2} / 4}(\Delta \omega), \quad \Delta \omega:=|K-1| \omega_{0} .
$$

The behavior $\operatorname{Imp}(\mathbf{M})=O(\Delta \omega)$ as $\Delta \omega \rightarrow 0$ is expected to be generic for any incoming pulse wavefunction that has spectrum sufficiently localized at $\omega_{0}$ with support (bandwidth) of size $\Delta \omega$. The precise prefactor that enters the formula for $\operatorname{Imp}(\mathbf{M})$ depends on the specifics of the pulse spectrum.

\subsection{Odd wavefunctions}

Next, we turn our attention to the equation $[8,11]$

$$
\mathrm{i} \partial_{t} \vec{\psi}=-\partial_{x}^{2} \vec{\psi}+\mathrm{iq} \delta_{p}^{\prime}(x)\left(\int_{-\infty}^{+\infty} \mathrm{d} y \delta_{p}^{\prime}(y) \vec{\psi}(y, t)\right),
$$

where $\delta_{p}^{\prime}(x)$ denotes $\delta^{\prime}(x)$ modified to remove any discontinuity at $x=0$ from the function on which it acts [8]: $\delta_{p}^{\prime}(x) g(x):=\delta^{\prime}(x)\left[1-\lim _{x \rightarrow 0^{+}}\right] g(x)$ for $x>0$ and $\delta_{p}^{\prime}(x) g(x):=\delta^{\prime}(x)\left[1-\lim _{x \rightarrow 0^{-}}\right] g(x)$ for $x<0$. For simplicity we write

$$
\vec{\psi}_{x}(0, t)=\int_{-\infty}^{+\infty} \mathrm{d} y \delta_{p}^{\prime}(y) \vec{\psi}(y, t)
$$

The incoming wavefunction is assumed to be the odd part in (29) [11]. The Fourier transform in $t$ of (81) gives

$$
\omega \vec{\phi}=-\partial_{x}^{2} \vec{\phi}-\mathbf{i q} \delta_{p}^{\prime}(x) \vec{\phi}_{x}(0, \omega) .
$$

By proposition I in section 2.2 and equation (23), the entanglement quaternion reads

$$
\Delta \mathbf{m}=\int_{-\infty}^{+\infty} \mathrm{d} t[\mathbf{n}(0, t), \mathbf{q}]
$$

where

$$
\mathbf{n}(0, t):=\mathrm{i} \vec{\psi}_{x}(0, t) \vec{\psi}_{x}^{\dagger}(0, t)
$$

By use of the Fourier transform of $\vec{\psi}(x, t)$, we have

$$
\Delta \mathbf{m}=\int_{0}^{+\infty} \frac{\mathrm{d} \omega}{2 \pi}[\widetilde{\mathbf{n}}(0, \omega), \mathbf{q}], \quad \widetilde{\mathbf{n}}(0, \omega)=\mathrm{i} \vec{\phi}_{x}(0, \omega) \vec{\phi}_{x}^{\dagger}(0, \omega) .
$$

The solution of (83) reads

$$
\vec{\phi}(x, \omega)=\vec{a}_{+}(\omega) \mathrm{e}^{\mathrm{i} \sqrt{\omega} x}+\vec{a}_{-}(\omega) \mathrm{e}^{-\mathrm{i} \sqrt{\omega} x}+\mathrm{i} \mathbf{q} G_{x}(x, \omega) \vec{\phi}_{x}(0, \omega),
$$

where $G_{x}$ is the derivative of the Green's function (39), i.e.,

$$
G_{x}(x, \omega)=-\frac{1}{4} \operatorname{sg}(x)\left(\mathrm{e}^{\mathrm{i} \sqrt{\omega} x}+\mathrm{e}^{-\mathrm{i} \sqrt{\omega} x}\right) .
$$

Note that the pseudo-potential $\delta_{p}^{\prime}$ gives zero when it acts on $G_{x}$ [8]. Thus, applying $\delta_{p}^{\prime}$ on (87) yields

$$
\vec{\phi}_{x}(0, \omega)=\mathrm{i} \sqrt{\omega}\left\{\vec{a}_{+}(\omega)-\vec{a}_{-}(\omega)\right\}
$$


Substitution of (89) into (87) with $\vec{d}=\left(\vec{d}_{+}, \vec{d}_{-}\right)^{T}$ leads to the formula

$$
\vec{d}(x, \omega)=\left[\begin{array}{cc}
\mathbf{1}+\frac{g}{4} \operatorname{sg}(x) \sqrt{\omega} \mathbf{u} & -\frac{g}{4} \operatorname{sg}(x) \sqrt{\omega \mathbf{u}} \\
\frac{g}{4} \operatorname{sg}(x) \sqrt{\omega} \mathbf{u} & \mathbf{1}-\frac{g}{4} \operatorname{sg}(x) \sqrt{\omega} \mathbf{u}
\end{array}\right]\left[\begin{array}{l}
\vec{a}_{+} \\
\vec{a}_{-}
\end{array}\right] .
$$

According to the imposed antisymmetry, we introduce the $2 \times 1$ vector $\vec{d}_{a}$ in

$$
\left[\begin{array}{l}
\vec{a}_{+} \\
\vec{a}_{-}
\end{array}\right]=\left[\begin{array}{c}
\vec{d}_{a} \\
-\vec{d}_{a}
\end{array}\right]
$$

Thus, we have

$$
\begin{aligned}
& \vec{d}_{+}(x, \omega)=\left\{1+\frac{g}{2} \operatorname{sg}(x) \sqrt{\omega} \mathbf{u}\right\} \vec{d}_{a}, \\
& \vec{d}_{-}(x, \omega)=-\left\{\mathbf{1}-\frac{g}{2} \operatorname{sg}(x) \sqrt{\omega} \mathbf{u}\right\} \vec{d}_{a} .
\end{aligned}
$$

Furthermore, by (87),

$$
\vec{\phi}_{x}(0, \omega)=2 \mathrm{i} \sqrt{\omega} \vec{d}_{a}
$$

The vector $\vec{d}_{\text {in }}$ corresponding to in-states can be $\vec{d}_{-}(+\infty, \omega)$ or $\vec{d}_{+}(-\infty, \omega)$; compare to (41). For example,

$$
\vec{d}_{\text {in }}(\omega)=\left(\mathbf{1}-\frac{g}{2} \sqrt{\omega} \mathbf{u}\right) \vec{d}_{a}
$$

Hence, by analogy with section 4.1 it makes sense to define

$$
\mathbf{1}+\frac{g}{2} \sqrt{\omega} \mathbf{u}=: p(\omega) \mathrm{e}^{\theta \mathbf{u}}
$$

where

$$
p(\omega)=\sqrt{1+\omega g^{2} / 4}, \quad \theta(\omega)=\arctan \left(\frac{g}{2} \sqrt{\omega}\right)
$$

Equation (86) for $\Delta \mathbf{m}$ becomes

$$
\Delta \mathbf{m}=\int_{0}^{+\infty} \frac{\mathrm{d} \omega}{2 \pi} \omega \frac{4 g}{p^{2}} \mathrm{e}^{\theta \mathbf{u}}\left[\mathrm{i} \vec{d}_{\text {in }} \vec{d}_{\mathrm{in}}^{\dagger}, \mathbf{u}\right] \mathrm{e}^{-\theta \mathbf{u}} .
$$

The assumption for a pure initial state amounts to using i $\vec{d}_{\text {in }} \vec{d}_{\text {in }}^{\dagger}$ from (71), i.e. i $\vec{d}_{\text {in }} \vec{d}_{\text {in }}^{\dagger}=$ $|f(\omega)|^{2} \mathbf{t}_{\text {in }}$, where $\mathbf{t}_{\text {in }}=\mathrm{i} \vec{s}_{\text {in }} \vec{s}_{\text {in }}^{\dagger}$ and $\vec{s}_{\text {in }}$ is introduced in (28). Note that

$$
\frac{\mathrm{d} \omega}{\mathrm{d} \theta}=\frac{4}{g} \sqrt{\omega} p^{2}
$$

Thus, by analogy with the symmetric case (section 4.1) we find the formula

$$
\Delta \mathbf{m}=16 \int_{0}^{+\infty} \frac{\mathrm{d} \omega}{2 \pi}\left\{\mathrm{e}^{\theta(\omega) \mathbf{u}}\left(P_{\mathbf{u}} \mathbf{t}_{\mathrm{in}}\right) \mathrm{e}^{-\theta(\omega) \mathbf{u}}\right\}\left(\omega^{3 / 2}|f(\omega)|^{2}\right)^{\prime} .
$$

The impurity $\operatorname{Imp}(\mathbf{M})$ follows by the procedure of section 4.1 .

Example of small-impurity pulse. A simple example of an incoming pulse wavefunction is described again by $(2 \pi)^{-1} \omega^{3 / 2}|f(\omega)|^{2}=H\left(\omega-\omega_{0}\right)-H\left(\omega-K \omega_{0}\right)$. The analysis for the impurity follows the steps of section 4.1 and is omitted here. Equation (79) should be recovered, where the angles $\theta_{1}$ and $\theta_{2}$ are now defined by

$\theta_{1}=\arctan \left(\frac{g \sqrt{\omega_{0}}}{2}\right), \quad \theta_{2}=\arctan \left(\frac{g \sqrt{\omega_{0} K}}{2}\right) ; \quad 0<\theta_{1}<\theta_{2}<\frac{\pi}{2}$. 


\section{Conclusion}

We introduced a general formulation of the nonrelativistic scattering from a two-state quantum memory in one-space dimension. The key feature is to view the interaction potential as an imaginary quaternion. In the case with point interactions, scattering from the memory amounts to a rotation in the frequency domain of an appropriately defined incoming quaternionic state.

We described the time evolution of the (entanglement) reduced density matrix in terms of the space integral of appropriate quaternionic commutators. By identifying quaternions with 4-vectors we point out that, because of the space integration, the quaternions involved in the entanglement evolution are timelike. Accordingly, the impurity measure for the final memory state is described by a time-integral containing the Minkowski norm of timelike, hyperbolic quaternions. In the special case of narrow-band pulse wavefunctions, the resulting impurity $\operatorname{Imp}(\mathbf{M})$ is generically of the order of the pulse bandwidth.

Our model based on scattering of particles from the memory differs from standard models where memory states correspond to quantum spins ('qubits'), e.g. [24, 25]. The basic difference lies in the fact that memory states in our formulation do not transform unitarily. Decoherence in our framework results from memory operations and not from unwanted couplings with the environment or dephasing noise [25]. However, our use of quaternions in the time-dependent Schrödinger equation with two coupled channels brings forth a geometric interpretation of scattering which is analogous to the action of the Lorentz group on qubit states [24]. Further study of this analogy is the subject of future work.

Our approach can be useful for addressing several questions. It is tempting to study rigorously successive scatterings from a quantum memory modeled by point interactions. This case is discussed for small impurities in [26] where it is concluded that impurities add up over a sequence of scatterings. The incoming states may not be pure but incoming quaternions are successively rotated in an appropriate sense in the frequency domain. An interesting question is how impurity is changed generally by this process. It is expected that $\operatorname{Imp}(\mathbf{M})$ always increases, especially if $\operatorname{Imp}(\mathbf{M})$ is thought of as 'entropy' in a sense [27]. There is no rigorous justification of this claim at the moment. The connection of $\operatorname{Imp}(\mathbf{M})$ to interference effects critical to quantum computing such as those discussed in [28] was not addressed by our analysis.

Another possible extension is the case of a $n$-state memory. A related issue is to define the appropriate algebra of $n \times n$ matrices that describe scattering in this context.

Finally, it is interesting to consider relativistic massive particles within the present framework. A starting point would be the case of particles with spin 1/2. A perhaps naive model problem is the one-dimensional scattering from a two-state memory in the setting of Dirac's equation. In this case, the particle-memory system is described by a $8 \times 1$ vector field. The study of this process by use of an analogous formalism is the subject of future work.

\section{Acknowledgment}

We thank John M Myers for invaluable discussions.

\section{Appendix. Proof of proposition I}

In this appendix, we prove proposition I of section 2.2. In particular, we show that an arbitrary null quaternion can be written as a tensor product of the form $\vec{\psi} \vec{\psi}{ }^{\dagger}$. Furthermore, we show that the impurity measure $\operatorname{Imp}(\mathbf{M})$ is given by (32). 
Suppose we have the null quaternion

$$
\mathbf{r}=\mathrm{i} r^{0} \sigma_{0}+\sum_{a=1}^{3} r^{a} \breve{\sigma}_{a}
$$

and let us write $\vec{r}:=\left(r^{1}, r^{2}, r^{3}\right)$ for the space vector. The 'nullity' property means that $\left(r^{0}\right)^{2}=|\vec{r}|^{2}$; we normalize so that $r^{0}=1 / 2$. The nullity is invariant under scaling. So, if we set $\mathbf{h}=2 \mathbf{r}$ we have that $\mathbf{h}$ is also null with $h^{0}=1$ and $|\vec{h}|=1$. An appropriate stereographic projection can identify the unit vector $\vec{h}$ with the complex number

$$
z:=\frac{h^{1}-\mathrm{i} h^{2}}{1-h^{3}}
$$

The inversion of this mapping yields

$$
r^{1}=\frac{\left(z+z^{*}\right) / 2}{1+z z^{*}}, \quad r^{2}=-\frac{\left(z-z^{*}\right) / 2 \mathrm{i}}{1+z z^{*}}, \quad r^{3}=\frac{\left(z z^{*}-1\right) / 2}{1+z z^{*}} .
$$

The substitution $z:=z_{1} / z_{2}$ into (A.3) where $\left|z_{1}\right|^{2}+\left|z_{2}\right|^{2}=1$ gives

$$
\begin{aligned}
r^{0} & =\left(z_{1} z_{1}^{*}+z_{2} z_{2}^{*}\right) / 2, & r^{1} & =\left(z_{1} z_{2}^{*}+z_{1}^{*} z_{2}\right) / 2, \\
r^{2} & =\left(z_{1}^{*} z_{2}-z_{1} z_{2}^{*}\right) / 2 \mathrm{i}, & r^{3} & =\left(z_{1} z_{1}^{*}-z_{2} z_{2}^{*}\right) / 2 .
\end{aligned}
$$

These relations show that an arbitrary null quaternion can be written as the tensor product $\vec{\psi} \vec{\psi}^{\dagger}$ where

$$
\vec{\psi}:=\left[\begin{array}{c}
z_{1} \\
z_{2}
\end{array}\right], \quad\left|z_{1}\right|^{2}+\left|z_{2}\right|^{2}=1 .
$$

Next, we show formula (32) for $\operatorname{Imp}(\mathbf{M})$. By setting $\mathbf{M}=-\mathrm{ir}$, it is straightforward to calculate

$$
\mathbf{r}^{2}=\left(-\left(r^{0}\right)^{2}-|\vec{r}|^{2}\right) \sigma_{0}+2 \mathrm{i} r^{0} \sum_{a} r^{a} \breve{\sigma}_{a}
$$

By invoking the algebra of the Pauli matrices, we find

$$
1-\operatorname{tr}\left(\mathbf{M}^{2}\right)=2\left[\left(r^{0}\right)^{2}-|\vec{r}|^{2}\right]=-2\left(\mathbf{r r}^{d}\right),
$$

so that

$$
\operatorname{Imp}(\mathbf{M})=\sqrt{2} \sqrt{\left|\mathbf{r r}^{d}\right|}=\sqrt{2} \sqrt{|\langle\mathbf{r}, \mathbf{r}\rangle|}=\sqrt{2}\|\mathbf{r}\|,
$$

which confirms (32) of proposition $\mathrm{I}$ in section 2.2. In the above, $\mathbf{r}=\left(r^{0}, r^{1}, r^{2}, r^{3}\right)$ is a four-component vector and $<\cdot, \cdot\rangle$ is the Minkowski inner product.

\section{References}

[1] Benioff P 1980 J. Stat. Phys. 22563

[2] Albert D Z 1983 Phys. Lett. A 98249

[3] Feynman R P 1985 Optics News 1111

[4] Deutch D 1985 Proc. R. Soc. Lond. Ser. A 40097

[5] Shor P W 1995 Phys. Rev. A 52 R2493

[6] Jaeger G 2006 Quantum Information: An Overview (Berlin: Springer)

[7] Heiss D 2002 Fundamentals of Quantum Information: Quantum Computation, Communication, Decoherence and All That (Berlin: Springer)

[8] Wu T T and Yu M L 2002 J. Math. Phys. 435949

[9] Wu T T 2003 Proc. SPIE on Quantum Information and Computation 5105 ed E Donkor, A R Pirich and H E Brandt (Bellingham, WA: SPIE Optical Engineering Press) 204 
[10] Wu T T 2005 Proc. SPIE on Quantum Information and Computation III 5815 ed E Donkor, A R Pirich and H E Brandt (Bellingham, WA: SPIE Optical Engineering Press) 62

[11] Margetis D and Myers J M 2006 J. Phys. A: Math. Gen. 3911567

[12] Hamilton W R 1969 Elements of Quaternions (New York: Chelsea)

[13] Weyl H 1952 Space-Time-Matter (New York: Dover)

[14] Naber G L 1992 The Geometry of Minkowski Spacetime (New York: Dover)

[15] Ward J P 1997 Quaternions and Cayley Numbers: Algebra and Applications (Boston: Kluwer)

[16] Macfarlane A 1900 Proc. R. Soc. Edinb. 23169

[17] Adler S L 1995 Quaternionic Quantum Mechanics and Quantum Fields (Oxford: Oxford University Press)

[18] Arrighi P and Patricot C 2003 J. Phys. A: Math. Theor. 36 L287

[19] Arrighi P and Patricot C 2003 Phys. Rev. A 68042310

[20] Shah J 1996 Ultrafast Spectroscopy of Semiconductors and Semiconductor Nanostructures (Berlin: Springer)

[21] Zanardi P and Rossi F 1998 Phys. Rev. Lett. 814752

[22] Dym H and McKean H P 1972 Fourier Series and Integrals (New York, NY: Academic)

[23] Masujima M 2005 Applied Mathematical Methods in Theoretical Physics (Weinheim, Germany: Wiley-VCH)

[24] Teodorescu-Frumosu M and Jaeger G 2003 Phys. Rev. A 67052305

[25] Ann K and Jaeger G 2007 Phys. Rev. B 75115307

[26] Myers J M and Wu T T 2007 Proc. SPIE on Quantum Information and Computation V 6573 ed E Donkor, A R Pirich and H E Brandt (Bellingham, WA: SPIE Optical Engineering Press) 657306

[27] Wootters W K 1998 Phys. Rev. Lett. 802245

[28] Jaeger G, Horne M A and Shimony A 1993 Phys. Rev. A 481023 Jaeger G, Shimony A and Vaidman L 1995 Phys. Rev. A 5154 